The Chittagong Univ. J. Sci. 41(1) : 106-111, 2019

DOI: https://doi.org/10.3329/cujs.v41i1.51918

\title{
On the Equation $Z(n)+S L(n)=n$
}

\author{
A. A. K. Majumdar ${ }^{1}$ and G. C. $\operatorname{Ray}^{2} *$ \\ 1. APU, 1-1 Jumonjibaru, Beppu-shi 874-8577, Japan \\ 2. Department of Mathematics, University of Chittagong, Chittagong-4331, \\ Bangladesh, E-mail: gcray07@yahoo.com \\ *Corresponding Author
}

\begin{abstract}
This paper considers the Diophantine equation $Z(n)+S L(n)=n$, where $Z(n)$ is the pseudo Smarandache function and $S L(n)$ is the Smarandache LCM function.

Keywords: Pseudo Smarandache function, Smarandache LCM function, Diophantine equation

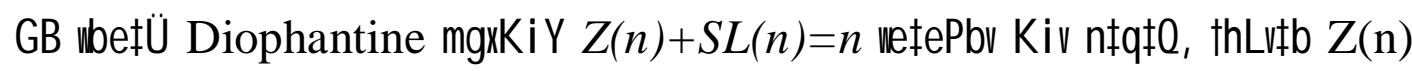

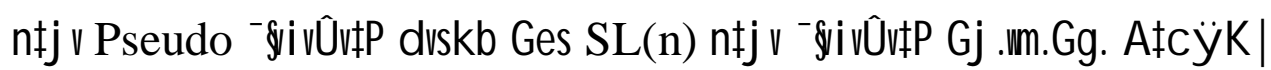

\section{Introduction}

The pseudo Smarandache function $Z(n)$, introduced by Kashihara [1], is defined as follows :

$$
Z(n)=\min \left\{m: n \mid \frac{\mathrm{m}(\mathrm{m}+1)}{2}\right\} .
$$

The Smarandache LCM function, denoted by $S L(n)$, is defined as

$$
\operatorname{SL}(\mathrm{n})=\min \{\mathrm{k} \geq 1: \mathrm{n} \mid[1,2, \ldots, \mathrm{k}]\},
$$

where $[1,2, \ldots, \mathrm{k}]$ is the least common multiple of the integers $1,2, \ldots, \mathrm{k}$. 
Then, we have the following result.

Lemma 1.1. Let $n=p_{1}{ }^{\alpha_{1}} p_{2}{ }^{\alpha_{2}} \ldots p_{r}{ }^{\alpha_{r}}$ be the (unique) representation of the integer $n$ in terms of its $\mathrm{r}$ prime factors $\mathrm{p}_{1}, \mathrm{p}_{2}, \ldots, \mathrm{p}_{\mathrm{r}}$. Then,

$$
S L(n)=\max \left\{\mathrm{p}_{1}^{\alpha_{1}}, \mathrm{p}_{2}^{\alpha_{2}}, \ldots, \mathrm{p}_{\mathrm{r}}{ }^{\alpha_{\mathrm{r}}}\right\} .
$$

In a paper, $\mathrm{Xin} \mathrm{Xu}[2]$ considers the Diophantine equation

$$
Z(n)+S L(n)=n \text {. }
$$

In this paper, we follow a simple approach to the solution of the above equation.

\section{Main Result}

Though a closed-form expression of $S L(n)$ is available, given in Lemma 1.1, the general form of $Z(n)$ has not yet been found. The expressions of $Z(n)$ for some particular cases of n, obtained so far, are given in Majumdar [3-5]. For a brief review of the pseudo Smarandache function, we refer the reader to Liu [6]. Theorem 4.2.2 in [3] gives a method of finding $Z(p q)$, where $p$ and $q(>p)$ are distinct primes. We follow the same method to find $Z\left(2^{k} p^{\alpha}\right)$ for some special cases. This is done in the lemma below.

Lemma 2.1. Let the integer $n$ be of the form

$$
n=2^{k} p^{\alpha}
$$

where $p \geq 3$ is a prime, and $k \geq 1$ and $\alpha \geq 1$ are integers. Then,

(1) if $2^{k+1} \mid\left(p^{\alpha}-1\right)$, then $Z(n)=p^{\alpha}-1$,

(2) if $2^{k} \mid\left(p^{\alpha}-1\right)$ but $2^{k+1}$ does not divide $\left(p^{\alpha}-1\right)$, then $Z(n)=p^{\alpha}\left(2^{k}-1\right)$,

(3) if $p^{\alpha} \mid\left(2^{k}-1\right)$, then $Z(n)=2^{k}\left(p^{\alpha}-1\right)$. 


\section{Proof. Since}

$$
Z(n)=Z\left(2^{k} p^{\alpha}\right)=\min \left\{m: 2^{k} p^{\alpha} \mid \frac{\mathrm{m}(\mathrm{m}+1)}{2}\right\},
$$

there are two possibilities :

Case 1. $2^{k+1}\left|m, p^{\alpha}\right|(m+1)$.

In this case,

$$
\begin{aligned}
& m=2^{k+1} x \text { for some integer } x \geq 1, \\
& m+1=p^{\alpha} y \text { for some integer } y \geq 1 .
\end{aligned}
$$

This leads to the following Diophantine equation :

$$
p^{\alpha} y-2^{k+1} x=1 \text {. }
$$

Case 2. $2^{k+1}\left|(m+1), p^{\alpha}\right| m$.

Then,

$$
\begin{aligned}
& m+1=2^{k+1} x \text { for some integer } x \geq 1, \\
& m=p^{\alpha} y \text { for some integer } y \geq 1,
\end{aligned}
$$

so that the resulting Diophantine equation is

$$
2^{k+1} x-p^{\alpha} y=1 .
$$

(1) Let $2^{k+1} \mid\left(p^{\alpha}-1\right)$. Then,

$$
p^{\alpha}-1=2^{k+1} a \text { for some integer } a \geq 1 .
$$

Then, the Diophantine equations (1) and (2) take the forms

$$
\begin{aligned}
& \left(2^{k+1} a+1\right) y-2^{k+1} x=1, \\
& 2^{k+1} x-\left(2^{k+1} a+1\right) y=1,
\end{aligned}
$$


that is,

$$
\begin{aligned}
& 2^{k+1}(a y-x)+y=1, \\
& 2^{k+1}(x-a y)-y=1 .
\end{aligned}
$$

Therefore, the minimum solution is obtained from (3) with

$$
y=1, \text { ay }-x=0 .
$$

Hence, the minimum $m$ is given by

$$
m=p^{\alpha}-1 \text {. }
$$

(2) Let $2^{k}$ divide $\left(p^{\alpha}-1\right)$ but $2^{k+1}$ does not divide $\left(p^{\alpha}-1\right)$. Then,

$$
p^{\alpha}-1=2^{k} b \text { for some integer } b \geq 1, b \neq 2 \text {. }
$$

Therefore, the Diophantine equations (1) and (2) can be written as

$$
\begin{aligned}
& \left(2^{k} b+1\right) y-2^{k+1} x=1, \\
& 2^{k+1} x-\left(2^{k} b+1\right) y=1,
\end{aligned}
$$

that is,

$$
\begin{aligned}
& 2^{k}(b y-2 x)+y=1, \\
& 2^{k}(2 x-b y)-y=1 .
\end{aligned}
$$

In this case, the minimum solution is obtained from (6) as follows:

$$
2 x-b y=1, y=2^{k}-1,
$$

Thus, the minimum $\mathrm{m}$ is

$$
m=p^{\alpha} y=p^{\alpha}\left(2^{k}-1\right) .
$$

(3) Let $p^{\alpha} \mid\left(2^{k}-1\right)$.

Here,

$$
2^{k}-1=p^{\alpha} c \text { for some integer } c \geq 1 .
$$

Then, the Diophantine equations (1) and (2) become 
110 On the Equation $Z(n)+S L(n)=n$

$$
\begin{aligned}
& p^{\alpha} y-2\left(p^{\alpha} c+1\right) x=1, \\
& 2\left(p^{\alpha} c+1\right) x-p^{\alpha} y=1,
\end{aligned}
$$

that is,

$$
\begin{aligned}
& p^{\alpha}(y-2 c x)-2 x=1, \\
& p^{\alpha}(2 c x-y)+2 x=1 .
\end{aligned}
$$

The minimum solution, obtained from (7) is

$$
y-2 c x=1,2 x=p^{\alpha}-1 .
$$

Consequently, the minimum $\mathrm{m}$ is

$$
m=2^{k+1} x=2^{k}\left(p^{\alpha}-1\right) .
$$

All these complete the proof of the lemma.

We now have the following theorem, giving the solution of the Diophantine equation

$$
Z(n)+S L(n)=n .
$$

Theorem 2.1. The Diophantine equation $Z(n)+S L(n)=n$ has the solution

$$
n=2^{k} p^{\alpha},
$$

where $2^{k}$ and $p^{\alpha}$ satisfy one of the two conditions (2) and (3) of Lemma 2.1

Proof. is evident from Lemma 2.1.

\section{Conclusions}

In the paper, we have obtained the solution of the Diophantine equation

$$
Z(n)+S L(n)=n \text { in the form } n=2^{k} p^{\alpha}
$$




\section{References}

[1] Kenichiro Kashihara: "Comments and Topics on Smarandache Notions and Problems”, Erhus University Press, U.S.A., 1996, 9, 48.

[2] Xin Xu: "An Equation Involving the Pseudo Smarandache Function and F. Smarandache LCM Function”, Scientia Magna, 2009, 5, 41.

[3] A. A. K. Majumdar: ProQuest, U.S.A., 2010, 7, 57.

[4] A. A. K. Majumdar: Jahangirnagar Journal of Mathematics and Mathematical Sciences, 2011, 26, 131.

[5] A. A. K. Majumdar: Scientia Magna, 2012, 8, 95.

[6] Liu Huaning: Scientia Magna, 2017, 12, 145.

Manuscript received on 20 December, 2018, Revised manuscript received on 19 August, 2019 and accepted on 20 August, 2019

The Chittagong Univ. J. Sc. Vol. 41(1), 2019 